\title{
Stochastic Resonance in Noisy Nondynamical Systems
}

\author{
J. M. G. Vilar, G. Gomila, and J. M. Rubí \\ Departament de Física Fonamental, Facultat de Física, Universitat de Barcelona, Diagonal 647, E-08028 Barcelona, Spain
} (Received 16 June 1997)

\begin{abstract}
We have analyzed the effects of the addition of external noise to nondynamical systems displaying intrinsic noise, and established general conditions under which stochastic resonance appears. The criterion we have found may be applied to a wide class of nondynamical systems, covering situations of different nature. Some particular examples are discussed in detail. [S0031-9007(98)06559-4]

PACS numbers: $05.40 .+\mathrm{j}$
\end{abstract}

Stochastic resonance (SR) [1-16] is a phenomenon wherein the addition of noise to a system enhances its response to a periodic input signal. This fact is usually characterized by an increase of the output signal-to-noise ratio (SNR) as the noise level increases. The main fingerprint of this constructive role played by noise is the appearance of a maximum in the SNR at a nonzero noise level, although recently new manifestations have been found such as multiple maxima [17] or a divergent SNR with the noise level [18]. In spite of the fact that at the beginning this phenomenon was restricted to bistable systems, nowadays it is known that there are different situations in which SR appears, as, for instance, in monostable, excitable, and nondynamical systems.

In regards to nondynamical systems, SR has been found only in threshold and thresholdlike devices $[13,19,20]$ and in the situation in which the output of the system consists of a random train of identical pulses, with the probability of a pulse generation exponentially depending on the input signal [21]. Nondynamical systems, however, encompass more general situations, of which many display intrinsic noise whose effects cannot systematically be neglected.

In this Letter we address precisely the problem of establishing general conditions under which SR appears in noisy nondynamical systems. We will show that the addition of external noise to the periodic input signal may give rise to the enhancement of the response of the system, therefore implying the presence of SR. In this sense, SR emerges due to the interplay between two noises, in such a way that the addition of external noise helps to overcome the limitations imposed by the unavoidable intrinsic noise.

A nondynamical system can be characterized by its output as a function of given input parameters which do not depend on the output. In general, those systems relaxing fast to their stationary state, in which the properties of the system are completely determined by some input parameters, may be considered as nondynamical systems. This very general requirement is responsible for the fact that nondynamical systems are frequently found in many different scientific areas, encompassing a wide variety of situations, including, to mention just a few, biological membranes, ionic channels, solid state diodes, quantum dots, and self-assembled molecular nanostructures. Usually, the output is a fluctuating quantity which is characterized by its mean value and correlation function. The characteristics of this intrinsic noise, to some extent, are determined by the state of the system, which in turns depends on the value of the input. This stochastic behavior is reflected in the class of systems described by

$$
I(t)=H(V)+\xi(t),
$$

where $I(t)$ and $V$ are the output and the input, respectively. The function $H(V)=\langle I(t)\rangle$ corresponds to the deterministic response and $\xi(t)$ represents the intrinsic noise. Usually the characteristic time scales of $\xi(t)$ are smaller than any other entering the system. Under these circumstances, its power spectrum for the range of frequencies of interest is flat and, consequently, $\xi(t)$ can be approximated by white noise with zero mean and correlation function $\langle\xi(t) \xi(t+\tau)\rangle=G(V) \delta(\tau)$ [22]. Here, the intensity of the fluctuations, $G(V)$, shows the dependence of the noise on the state of the system. Equation (1) then describes, in general, the behavior of most noisy nondynamical systems. For instance, in the case of a linear resistor the output, $I$, is the current intensity with mean value given by the Ohm law, $H(V)=V / R$. The input, $V$, is the applied voltage and $R$ is the resistance. The noise term $\xi(t)$, accounting for thermal fluctuations and given by the Nyquist theorem, has a flat spectrum and intensity given by $G(V)=2 k T / R$. Here $k$ is the Boltzmann constant and $T$ is the absolute temperature of the resistor.

In order to analyze how the addition of external noise affects the response of the system to a slow periodic signal, we consider the input consisting of sinusoidal and random contributions, namely, $V \equiv V(t)=V_{s}(t)+$ $V_{r}(t)$. Here $V_{s}(t)=\alpha \sin \left(\omega_{0} t\right)$, with $\alpha$ being the amplitude and $\omega_{0} / 2 \pi$ the frequency. The random term $V_{r}(t)$ is assumed to be stationary Gaussian noise with zero mean and correlation function $\left\langle V_{r}(t) V_{r}(t+\tau)\right\rangle=$ $\sigma^{2} \exp \left(-\tau / \tau_{F}\right)$, where $\sigma^{2}$ defines the noise level and $\tau_{F}$ is the correlation time, which is assumed $\tau_{F} \ll 2 \pi / \omega_{0}$. The SNR can be computed from the averaged power 
spectrum

$$
\begin{aligned}
P(\omega)= & 4 \int_{0}^{2 \pi / \omega_{0}} \frac{\omega_{0}}{2 \pi} d t \\
& \times \int_{0}^{\infty}\langle I(t) I(t+\tau)\rangle_{\xi, r} \cos (\omega \tau) d \tau,
\end{aligned}
$$

which follows from the correlation function

$$
\begin{aligned}
\langle I(t) I(t+\tau)\rangle_{\xi, r}= & \langle H(V(t)) H(V(t+\tau))\rangle_{r} \\
& +\langle G(V(t))\rangle_{r} \delta(\tau) .
\end{aligned}
$$

Here $\langle.\rangle_{\xi, r}$ and $\langle.\rangle_{r}$ indicate average over both noises and only over $V_{r}(t)$, respectively. By considering sufficiently small amplitudes of the input signal and low noise level, $H(V)$ and $G(V)$ can be expanded in power series of $V$; therefore

$$
\mathrm{SNR}=\frac{2 \pi \alpha^{2} H^{\prime}\left(H^{\prime}+H^{\prime \prime \prime} \sigma^{2}\right)}{2 G+\left(4 \tau_{F} H^{\prime 2}+G^{\prime \prime}\right) \sigma^{2}},
$$

provided that $H^{\prime} \neq 0$. The symbol ' indicates the derivative of the function with respect to its argument [24]. Notice that all the functions are evaluated at $V=0$.

If $H^{\prime} \neq 0$ and the inequality

$$
2 H^{\prime \prime \prime} G-4 \tau_{F} H^{\prime 3}-H^{\prime} G^{\prime \prime}>0
$$

holds, the SNR is an increasing function of the noise level, which reveals that the addition of noise enhances the response of the system to a weak periodic signal. As usually occurs, if the SNR decreases for high noise level, then the SNR presents at least a maximum, thus indicating the appearance of SR. Notice that the previous inequality is a sufficient condition for the appearance of SR. In fact, when the approximation of low noise level is not valid, the SNR might be an increasing function of the noise level despite inequality (5) does not hold.

As a first example illustrating the applicability of our results, we will analyze the particular case discussed in Ref. [21] in our context. In the case of systems consisting of spike trains with Poisson statistics it is a well known result that the pulse generation rate gives the mean value of the process $H(V)$, as well as the intensity of the fluctuations $G(V)$ (see, for instance, Ref. [23], p. 314); therefore one has $G(V)=H(V)$. Under the circumstances of Ref. [21], $G(V)=H(V)=r(0) \exp (V)$, where $r(0)$ is the pulse generation rate at $V=0$. Then, Eq. (5) indicates that SR appears for $r(0) \tau_{F}<1 / 4$, in agreement with Refs. [21,25]. Note that for small amplitudes of the input signal, following our approach [Eqs. (1), (2), and (3)], the SNR can analytically be computed giving the same result as the one of Refs. [21,25].

In order to show the great generality of Eq. (5) we will analyze a system in which the internal noise does not follow Poisson statistics, as is the case, for instance, of a model for electrical conduction which displays saturation. In this model, $I(t)$ corresponds to the current intensity and
$V$ to an input voltage. To be explicit, we will consider

$$
\begin{gathered}
H(V)=\frac{V+V_{0}}{R\left[1+\left(V+V_{0}\right)^{2}\right]^{1 / 2}}, \\
G(V)=\frac{Q}{\left[1+\left(V+V_{0}\right)^{2}\right]^{1 / 2}},
\end{gathered}
$$

where $R$ and $Q$ are constants. Here $V_{0}$ represents a constant bias voltage. For low values of the total applied voltage $\left(V+V_{0}\right)$, the system behaves as a linear resistor. For high values, however, the nonlinear terms become important to the extent that the current intensity saturates. Semiconductor systems displaying hot electrons effects constitute a well known example of systems exhibiting this nonlinear behavior [26]. According to our previous analysis [Eq. (5)], this system exhibits SR for sufficiently large values of $V_{0}$. In Fig. 1 we have depicted the SNR as a function of the noise level, for different values of $V_{0}$. These results have been obtained by numerically averaging over realizations of the noise terms. SR appears for sufficiently large values of $V_{0}$, as predicted by the criterion established in Eq. (5). The occurrence of SR is not exclusive of this particular model. We have analyzed other models exhibiting saturation [27] and found that SR is a common phenomenon.

Let us now discuss the case in which $H^{\prime}=0$. In this situation, the SNR vanishes for $\sigma^{2}=0$. Therefore, for low noise level and sufficiently small amplitudes of the input signal, if $H(V)$ is not symmetric around $V=0$, the SNR must be an increasing function of $\sigma^{2}$; explicitly [28]

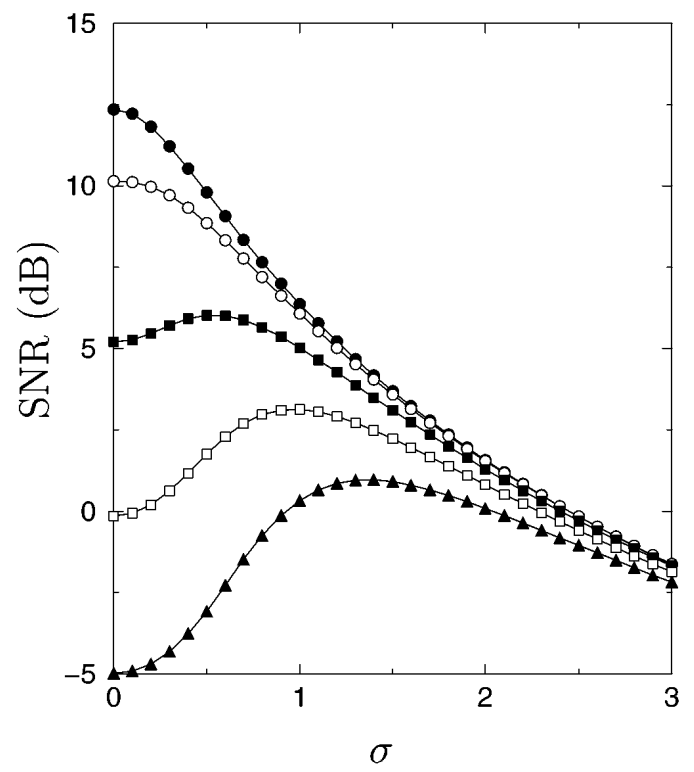

FIG. 1. SNR for the system described through Eqs. (6) and (7) as a function of $\sigma$ for different voltages: $V_{0}=0$ (filled circles), $V_{0}=0.5$ (empty circles), $V_{0}=1.0$ (filled squares), $V_{0}=1.5$ (empty squares), and $V_{0}=2$ (filled triangles). The values of the remaining parameters are $Q=0.1, R=1, \tau_{F}=$ $0.1, \alpha=0.32$, and $\omega_{0} / 2 \pi=0.1$. 


$$
\mathrm{SNR}=\frac{\pi \alpha^{2} H^{\prime \prime \prime 2} \sigma^{4}}{4 G} .
$$

One then concludes that, when $H^{\prime}=0, \mathrm{SR}$ is always present for sufficiently small amplitudes of the input signal and its appearance does not depend on the form of noise $\xi(t)$, provided that the noise term is different from zero at $V=0$. This situation may occur in many systems of interest, as, for instance, in tunnel diodes [27,29], ionic channels [30], and in a general way in any system exhibiting maxima, minima, or inflection points in the $I-V$ characteristics.

As an example of a system for which $H^{\prime}=0$, that can be solved analytically, for any value of the amplitude and noise level, we will consider the case

$$
\begin{aligned}
& H(V)=V^{3}, \\
& G(V)=Q,
\end{aligned}
$$

where $Q$ is a constant. The SNR is found to be

$$
\mathrm{SNR}=\pi \frac{18 \sigma^{4} \alpha^{2}+9 \sigma^{2} \alpha^{4}+\frac{9}{8} \alpha^{6}}{2 Q+\tau_{F}\left(44 \sigma^{6}+54 \sigma^{4} \alpha^{2}+\frac{27}{2} \sigma^{2} \alpha^{4}\right)} .
$$

From the previous result we can elucidate how the SNR behaves in all the range of values of $\sigma^{2}$ and $\alpha^{2}$. It is interesting to point out that for sufficiently small amplitudes of the input signal and low noise level $(\alpha \ll$ $\sigma^{2} \ll 1$ ), Eq. (11) reduces to Eq. (8), then the SNR $\sigma^{4}$ is an increasing function of $\sigma^{2}$ and SR appears, as predicted by our previous general analysis. For high noise level the behavior is SNR $\sim \sigma^{-2}$, then it decreases when increasing the noise level. Another notable feature is that the SNR is an increasing function of the noise level when $\tau_{F}$ is sufficiently small, irrespective of the value of the amplitude of the input signal. In Fig. 2 we have depicted

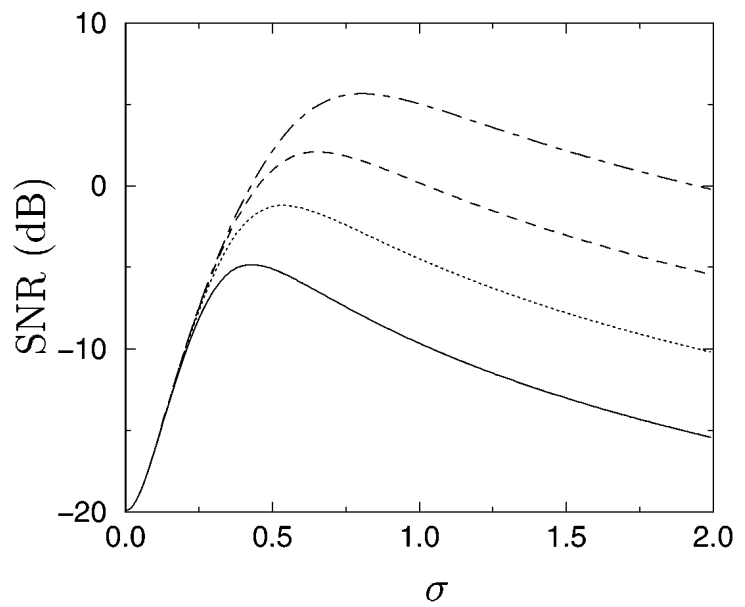

FIG. 2. SNR [Eq. (11)] for the system described through Eqs. (9) and (10) as a function of $\sigma$ for different correlation times of the input noise. The values of the parameters are $Q=0.1, \alpha=0.3, \tau_{F}=1$ (continuous line), $\tau_{F}=0.3$ (dotted line), $\tau_{F}=0.1$ (dashed line), and $\tau_{F}=0.03$ (dot-dashed line). the SNR corresponding to Eq. (11) as a function of the noise level, for different values of $\tau_{F}$. This figure clearly illustrates the appearance of a maximum in the SNR at nonzero noise level and the dependence of the SNR on the correlation time of the input noise, namely, the output of the system is enhanced when decreasing $\tau_{F}$.

Another interesting situation in which $H^{\prime}=0$ occurs is when the $I-V$ curve exhibits a plateau, as, for instance, in the case of a quantum dot displaying Coulomb gap [31]. Under these circumstances, an input of finite amplitude is required before any effect on the output is produced. Added noise helps small deterministic signals to reach this finite amplitude. Therefore, the output may be enhanced giving rise to the appearance of SR.

Finally, in order to elucidate how the addition of noise enhances the output in the class of systems we are considering, we have shown in Fig. 3 two time series corresponding to zero and the optimum noise levels for the explicit case given through Eqs. (9) and (10). This figure clearly shows that the addition of an optimal amount of noise makes the presence of the input signal manifest.
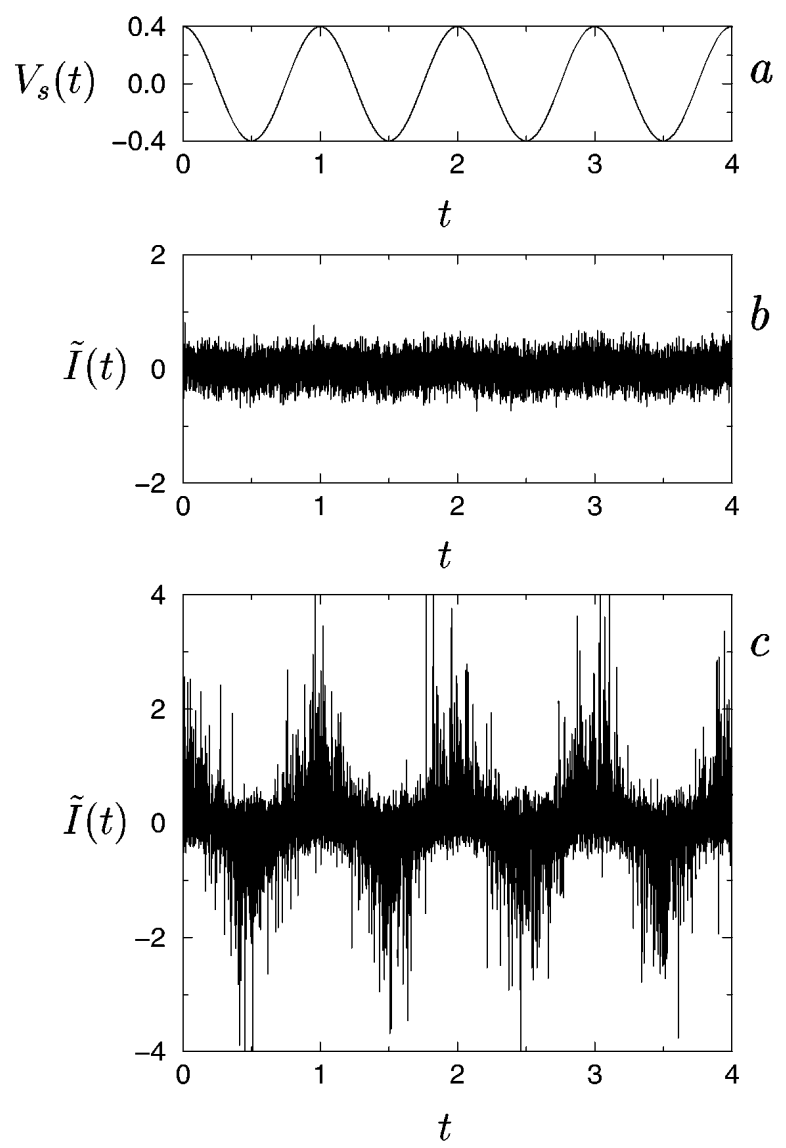

FIG. 3. (a) Input periodic signal. (b) Time evolution of $\tilde{I}(t)=\frac{1}{\Delta t} \int_{t}^{t+\Delta t} I(s) d s$ corresponding to Eqs. (9) and (10), with $\alpha=0.4, \quad \omega_{0} / 2 \pi=1, Q=10^{-5}, \Delta t=2.4 \times 10^{-4}$, $\tau_{F}=10^{-4}$, and $\sigma^{2}=0$. (c) Same situation as in (b) but $\sigma^{2}=0.4$. 
Our analysis has established general conditions in which SR emerges in noisy nondynamical systems. In this way, we have shown that the appearance of SR in nondynamical systems is not a particular situation occurring in threshold (or thresholdlike) devices and spiketrain systems with exponential rate generation, as usually believed. On the contrary, SR is a robust phenomenon that may occur in many different physical, chemical, and biological nondynamical systems. One aspect we emphasize is that a simple expression we have found is sufficient to make the presence of SR manifest. This general criterion, which refers to a wide class of nondynamical systems, may directly be applied to new cases, thereby extending the scope and perspectives of SR.

This work was supported by DGICYT of the Spanish Government under Grant No. PB95-0881. J. M. G. V. and G. G. thank Generalitat de Catalunya for financial support.

[1] R. Benzi, A. Sutera, and A. Vulpiani, J. Phys. A 14, L453 (1981).

[2] S. Fauve and F. Heslot, Phys. Lett. 97A, 5 (1983).

[3] B. McNamara, K. Wiesenfeld, and R. Roy, Phys. Rev. Lett. 60, 2626 (1988).

[4] L. Gammaitoni, F. Marchesoni, E. Menichella-Saetta, and S. Santuchi, Phys. Rev. Lett. 62, 349 (1989).

[5] B. McNamara and K. Wiesenfeld, Phys. Rev. A 39, 4854 (1989).

[6] A. Longtin, A. Bulsara, and F. Moss, Phys. Rev. Lett. 67, 656 (1991).

[7] Proceedings of the NATO Advanced Research Workshop on Stochastic Resonance, San Diego, 1992, edited by F. Moss, A. Bulsara, and M. F. Shlesinger [J. Stat. Phys. 70, 1 (1993)].

[8] J. K. Douglass, L. Wilkens, E. Pantazelou, and F. Moss, Nature (London) 365, 337 (1993).

[9] F. Moss, in Some Problems in Statistical Physics, edited by G. H. Weiss (SIAM, Philadelphia, 1994).

[10] K. Wiesenfeld, D. Pierson, E. Pantazelou, C. Dames, and F. Moss, Phys. Rev. Lett. 72, 2125 (1994).

[11] K. Wiesenfeld and F. Moss, Nature (London) 373, 33 (1995).

[12] J. F. Lindner, B. K. Meadows, W. L. Ditto, M. E. Inchiosa, and A. R. Bulsara, Phys. Rev. Lett. 75, 3 (1995).

[13] Z. Gingl, L. B. Kiss, and F. Moss, Europhys. Lett. 29, 191 (1995).

[14] M. Grifoni and P. Hanggi, Phys. Rev. Lett. 76, 1611 (1996).
[15] F. Marchesoni, L. Gammaitoni, and A. R. Bulsara, Phys. Rev. Lett. 76, 2609 (1996)

[16] J. M. G. Vilar and J. M. Rubí, Phys. Rev. Lett. 78, 2886 (1997).

[17] J. M. G. Vilar and J. M. Rubí, Phys. Rev. Lett. 78, 2882 (1997).

[18] J. M. G. Vilar and J. M. Rubí, Phys. Rev. Lett. 77, 2863 (1996).

[19] F. Chapeau-Blondeau and X. Godivier, Phys. Rev. E 55, 1478 (1997).

[20] Reference [19] shows the appearance of SR in a wide variety of nondynamical systems, including some without strict thresholds but with sufficiently steep parts. In this sense, however, SR appears when the system shares the main characteristics of a threshold device, i.e., a fast increasing of the output for a narrow range of values of the input.

[21] S. M. Bezrukov and I. Vodyanoy, Nature (London) 385, 319 (1997).

[22] We stress that this form of the noise term is quite general for sufficiently small frequencies, since if the power spectrum is flat the noise must be delta correlated as follows from the identity $\int_{-\infty}^{\infty} e^{i \omega \tau} d \omega=2 \pi \delta(\tau)$ [23].

[23] A. Papoulis, Probability, Random Variables, and Stochastic Processes (McGraw-Hill, New York, 1991), 3rd ed.

[24] The previous results can directly be generalized to the case in which the correlation function of the external noise is not exponential, i.e., $\left\langle V_{r}(t) V_{r}(t+\tau)\right\rangle=\sigma^{2} f(\tau)$, with $f(0)=1$, by replacing by $\tau_{F}$ by $\int_{0}^{\infty} f(\tau) d \tau$.

[25] Note that there is a numerical factor missing in Eqs. (7) and (8) of Ref. [21]. If this factor is taken into account, the condition for the appearance of SR obtained in Ref. [21] coincides with our condition.

[26] Hot Electron Transport in Semiconductors, edited by L. Reggiani (Springer-Verlag, Berlin, 1985).

[27] A. van der Ziel, Noise in Solid State Devices and Circuits (John Wiley \& Sons, New York, 1986).

[28] For the case in which the $n$ first odd derivatives of $H(V)$ vanish, the corresponding SNR is found to be SNR = $2 \pi \frac{\alpha^{2}}{2 G}\left[H^{(2 n+1)} \frac{1}{n !}\left(\frac{1}{2} \sigma^{2}\right)^{n}\right]^{2}$, where $H^{(2 n+1)}$ is the $(2 n+1)$ th derivative. The fact that $H(V)$ is nonsymmetric ensures that not all the odd derivatives of $H(V)$ vanish. It is worth realizing that when $H^{\prime}(V)=0$, the lowest order approximation in the noise level for the SNR does not depend on the correlation time of the noise $\tau_{F}$.

[29] S. M. Sze, Physics of Semiconductor Devices (John Wiley \& Sons, New York, 1981).

[30] E. Stefani et al., Proc. Natl. Acad. Sci. U.S.A. 94, 5427 (1997).

[31] R. P. Andres et al., Science 272, 1323 (1996). 